\title{
Virtual Reality as a Non-Pharmacological Adjunct to Reduce the Use of Analgesics in Hospitals
}

\author{
Shunn Theingi ${ }^{1}$ (D) $\cdot$ Ian Leopold ${ }^{2}$ (I) $\cdot{\text { Tolulope } \text { Ola }^{3} \text { (D) } \cdot \text { Gary S. Cohen }}^{2}$ (i) $\cdot$ Hillel S. Maresky ${ }^{2}$ (D)
}

Received: 29 December 2020 / Accepted: 26 March 2021 / Published online: 6 April 2021

(C) The Author(s), under exclusive licence to Springer Nature Switzerland AG 2021

\begin{abstract}
Virtual reality (VR) has become an increasingly viable non-pharmacological adjunct to reduce the use of analgesics in hospitals. Within the context of the ongoing opioid epidemic, VR can serve as an invaluable alternative to traditional pain management techniques. While VR research continues to advance, there is no clear consensus on the terms used to describe critical aspects of VR. This paper presents how immersion, presence, and agency encapsulate the VR experience and investigates the methods by which VR can relieve pain by changing users' cognition and perception of pain. Multiple clinical studies investigating VR efficacy indicate that higher degrees of immersion, presence, and agency are all correlated with greater pain reduction. These studies also demonstrate that VR analgesia is effective for patients with various medical conditions and for those undergoing painful medical procedures. Furthermore, the shared biological mechanisms between pain and anxiety suggest that reducing either through the use of VR will reduce the other. As a nascent field of research, VR analgesia has key obstacles to overcome in order to become a mainstream intervention for pain management in hospital settings.
\end{abstract}

Keywords Virtual reality $\cdot$ Analgesic $\cdot$ Perception $\cdot$ Cognition $\cdot$ Pain

\section{Introduction}

The opioid epidemic is an ongoing public health crisis with devastating consequences in the USA. Nearly 500,000 people in the USA have died from an overdose involving either prescription or illicit opioids between 1999 and 2019. In fact, over $70 \%$ of the 70,630 overdose deaths in 2019 involved an opioid, and over 136 people a day die from opioid-related drug overdoses (Centers for Disease Control and Prevention, 2021). The Centers for Disease Control and Prevention (CDC) estimates the total economic burden from prescription opioid misuse alone in the USA to be approximately $\$ 78.5$ billion a year, accounting for the costs of healthcare, lost productivity,

Shunn Theingi

shunntheingi@gmail.com

1 Lewis Katz School of Medicine, Temple University, Philadelphia, PA, USA

2 Department of Radiology, Lewis Katz School of Medicine, Temple University, Philadelphia, PA, USA

3 Hospital for Sick Children, University of Toronto, Ontario, Canada addiction treatment, and criminal justice (Florence et al., 2016). Opioids are the most potent analgesics that doctors can prescribe. An analysis of 2016 National Health Interview Survey data by the CDC reveals that an estimated $20.4 \%$ (50 million) of US adults had chronic pain and $8.0 \%$ (19.6 million) had high-impact chronic pain. Chronic pain was considered high-impact when it frequently limits activities of daily living (Dahlhamer et al., 2018). In 2018, over 168 million opioid prescriptions were dispensed in the USA (Centers for Disease Control and Prevention, 2019).

When utilizing opioids for pain management, therapeutic effects can be transient and ineffective, especially over longer treatment times. This can be partially explained by the multifaceted nature of pain, which consists of sensory, affective, and cognitive components, and is mediated by various brain regions and influenced by the integration of multiple inputs to generate nerve impulses that elicit pain (Triberti et al., 2014). Opioids also have adverse effects, such as increased pain sensitivity, constipation, nausea, and vomiting, which limit their dosage. There is also a need to reduce opioid analgesic use during medical procedures for patients with a history of opioid use disorder, as with long-term use, tolerance and physical dependence become increasingly likely. Fighting the opioid epidemic requires alternative pain management strategies. 
Virtual reality (VR) has recently emerged as a promising non-pharmacological adjunct analgesic in multiple treatment applications. VR allows users to immerse themselves and engage with a 3-dimensional computer-generated world. A VR setup typically includes VR software, head-mounted displays (HMDs), headphones, sensory input devices, motion tracking systems, and devices, like a computer mouse or game controller, to interact with or manipulate the virtual environment (Fig. 1). VR interventions have become increasingly accessible tools for pain management due to rapid technological advances and declining costs (Indovina et al., 2018). In this article, we discuss the key terms associated with VR, the viability of VR to reduce pain/anxiety, the key findings to date, and future directions.

\section{Immersion, Presence, and Agency}

Immersion, presence, and agency are three interdependent terms associated with describing aspects of the VR experience (Fig. 2). Because the field of research involving VR is still relatively new, there is no clear consensus on the terminology and researchers tend to use them differently. This section will put forward clear definitions of the terms and describe how changing immersion, presence, and agency can impact the VR experience.

Immersion is the illusion of feeling physically and psychologically immersed in a different environment. Although the degree of immersion is partially user-dependent, it is also contingent on objective factors such as the technical specifications of the VR system's hardware and software. An immersive VR experience will isolate and elicit the user's visual, auditory, and haptic senses such that it is simulating a tangible virtual environment (VE) with multi-sensory engagement. A greater degree of immersion enhances the distractive properties of VR to reduce pain and anxiety in burn patients (Hoffman et al., 2000). Increasing immersion tends to lead to a higher sense of presence.

Presence, a measure of how integrated the user feels in the $\mathrm{VE}$, is more subjective because it depends on both the user and the context of the VR experience. For example, the user's current mood, disposition towards VR, and past experiences with VR all affect how assimilated the user is to the VE.

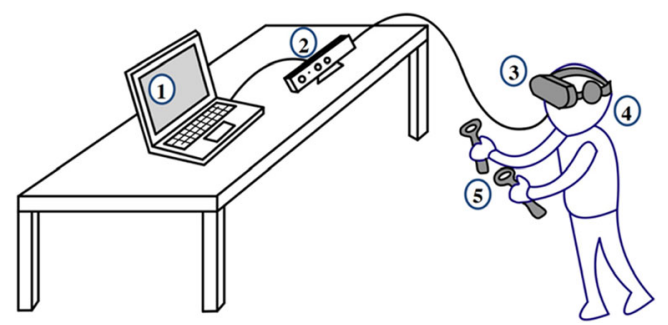

Fig. 1 VR setup with laptop (1), motion tracking system (2), headmounted display (3), headphones (4), and game controllers (5)

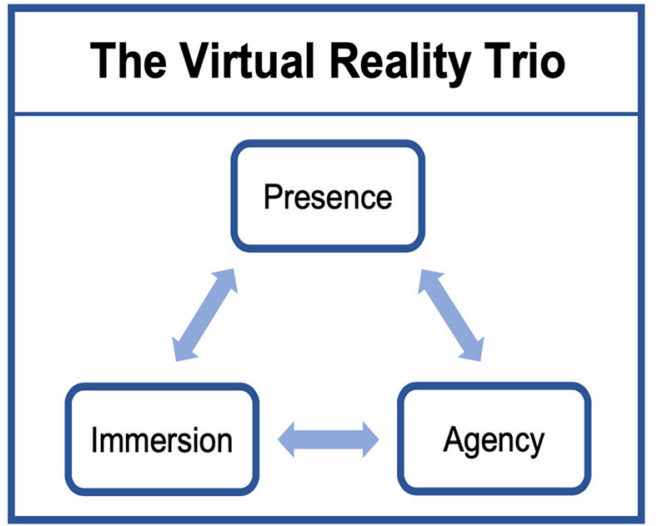

Fig. 2 Interdependence of immersion, presence, and agency

Children and adults require different VEs to induce enough presence for a convincing VR experience because children are less able to effectively separate fantasy and reality (Won et al., 2017). Multiple studies have shown that a higher sense of presence is correlated with greater pain reduction (GutierrezMartinez et al., 2010; Hoffman et al., 2000; Hoffman, Patterson, et al., 2004a; Hoffman, Sharar, et al., 2004c; Sharar et al., 2016; Won et al., 2017).

Agency refers to the interactivity of the VR system. The user has the autonomy to choose how to interact and engage with the VR; in return, the VE responds to the user's actions. This interactivity between user and system reduces cognitive dissonance and tricks the brain into believing that the VE is more realistic. Agency is the main distinguishing feature between 360-degree videos and true VR. While users can only passively watch 360 -degree videos, they can actively interact with true VR. Various studies showed that VR with more agency were more effective at relieving pain (Al-Ghamdi et al., 2020; Gutierrez-Martinez et al., 2010; Law et al., 2011; Wender et al., 2009). Agency is also associated with inducing a higher sense of presence, which is correlated with greater pain reduction.

\section{Changing Perception Through VR}

Pain perception has a strong psychological component. There have been a few theories proposed regarding the mechanism by which VR changes pain perception so that users experience reduced pain intensity. Melzack and Wall developed the gate control theory, which states that nociceptive signals have to pass through a "gate" in the spinal cord before reaching the brain. The intensity of pain perceived by the brain depends on a combination of sensory, behavioral, and psychological factors that influence the degree to which the gate is open or closed (Melzack \& Wall, 1965). The neuromatrix theory of pain broadly agrees with the gate control theory, but states that pain production originates 
from the central nervous system rather than tissue damage and the peripheral nervous system (Melzack, 1999). The biopsychosocial model of pain proposes that pain is a combination of sensory, behavioral, and psychological factors mediated by various brain regions (Triberti et al., 2014). All three theories suggest that the brain and its higher order thought processes play a crucial role in pain perception by altering how the individual interprets incoming pain signals and possibly changing the amount of pain signals that reach the brain (Hoffman et al., 2007; Hoffman, Richards, et al., 2004b). This section explores how VR can relieve pain by changing perceptions.

While opioids attenuate pain perception by disrupting the transmission of neural nociceptive signals to the central nervous system, VR changes pain perception by altering sensory, behavioral, and psychological factors. Although the exact mechanism through which VR functions to reduce pain remains unknown, most proposed mechanisms attribute the impact of VR to active distraction (Li et al., 2011). Higher levels of immersion, presence, and agency contribute to making the illusion of the VE more real, which enhances the ability of VR to alter a person's perception. The VE diminishes the pain by taking up finite attentional resources with non-painful neural signaling, leaving fewer resources for pain processing, and distracting the brain from nociceptive pain signals (Ahmadpour et al., 2019; Pourmand et al., 2018). VR distracts and changes patients' thoughts, which subsequently modifies their feelings and behaviors. The shift in their feelings and behaviors away from pain and anxious behavior, respectively, can further affect their thoughts and place them at ease. VR can also help patients acclimate and feel more comfortable with upcoming treatments and procedures. A study investigating the effect of a mock MRI service on pediatric patients undergoing general anesthesia (GA) for MRIs found that mock MRI reduced the need for GA (Carter et al., 2010). Similarly, VR and its endless possibilities for personalization can change individuals' perceptions of upcoming procedures and possibly reduce the need for anesthesia.

Cognitive behavioral therapy (CBT) is the prevailing psychotherapeutic treatment for patients with chronic pain. It was originally developed to treat patients with depression or anxiety disorders but has been effectively applied to a wide range of psychophysical disorders. CBT is based on the assumption that habitual thoughts and beliefs can influence patients' behaviors in ways that are harmful or beneficial. The goal of CBT is to learn how to identify maladaptive thought patterns, assess whether they are accurate depictions of reality, and apply personal coping strategies to generate constructive thought patterns. CBT empowers patients with selfmanagement strategies like relaxation and pacing so that they have more control over their perception of pain (Kerns et al., 2011). Administering CBT through VR can further enhance CBT's effectiveness in pain reduction by diverting attention away from nociceptive signals so that the perception of pain is even more diminished.

\section{Interplay Between Pain and Anxiety}

Anxiety disorders and chronic pain frequently coexist. They have a reciprocal relationship and tend to exacerbate each other-high anxiety can increase sensitivity to pain, and pain can increase anxiety-because of their shared biological mechanisms. The somatosensory cortex interacts with the amygdala, hypothalamus, and the anterior cingulate cortex to produce the psychological and physical experience of pain, but these anatomical brain regions also contribute to feelings of anxiety. Additionally, both fear and anxiety involve two neurotransmitters, serotonin and norepinephrine, which signal to the brain and nervous system in times of stress. This section will discuss the relationship between pain and anxiety and how VR can attenuate them.

Preoperative anxiety is prevalent among pediatric and adult patients undergoing surgical procedures. A low level of anxiety is an understandable reaction to the unpredictable and potentially hazardous nature of surgical procedures. However, higher levels of preoperative anxiety require larger doses of anesthetics and cause postoperative pain in the form of psychological and physiological stress, surgical complications, and a painful and prolonged recovery (Kain et al., 2006). One way to reduce preoperative anxiety is to provide information on what to expect before, during, and after the procedure. In one randomized clinical trial (RCT), the group of pediatric patients receiving the information through a 360-degree video had significantly lower preoperative anxiety and increased adherence during anesthesia than the control group of pediatric patients receiving conventional information regarding anesthesia and surgery (Ryu et al., 2017). In a different RCT of 127 patients undergoing cranial and spinal operations, a 5-min VR video through VR goggles describing the preoperative and postoperative experience improved preoperative anxiety and stress compared to a standard preoperative procedure (Bekelis et al., 2017). A study assessing the impact of VR on postoperative pain in adult patients within $24 \mathrm{~h}$ of their cardiac surgery found that a majority of the patients reported a significant pain reduction post-VR therapy (Mosso-Vázquez et al., 2014). In general, patients reporting less anxiety during VR were more likely to report pain reduction (Pourmand et al., 2018; Sharar et al., 2016).

The ability of VR to remove patients from the anxietyinducing clinical environment and immerse them in a VE reduces pain and anxiety in patients undergoing burn care, cancer procedures and treatments, and even routine medical 
procedures. In all of those instances, Li et al. (2011) found that VR intervention was able to effectively reduce both the associated pain and anxiety.

\section{VR as an Effective Analgesic}

Virtual reality has been shown to be an effective therapy for patients experiencing pain from various medical conditions and medical procedures. In the last two decades, VR has found many applications in healthcare, including pain management. This section evaluates the potential of VR to effectively reduce pain.

VR analgesia has been extensively studied in burn patients as their pain is often undertreated and tends to vary in intensity throughout their recovery. Managing severe burns requires daily wound dressing changes and physical therapy (PT), both of which can be painful. Out of the 22 clinical studies on burn patients that Indovina et al. (2018) assessed, all but one of the studies showed that VR decreased the pain experienced by pediatric and adult burn patients undergoing wound care and PT in contrast with the control conditions; in the one negative study, failure might be attributed to the fact that the VR group did not have agency in their VR, as defined above. Although the pain measurement tools varied in those 21 clinical studies, they reported that VR can significantly reduce pain in burn patients.

VR analgesia has also been assessed in patients with cancer undergoing chemotherapy, which frequently results in adverse effects, such as nausea, vomiting, fatigue, pain, depression, and anxiety. These side effects can result in dosage reduction or treatment discontinuation and ultimately decrease the likelihood of recovery. Patients with cancer also frequently undergo other painful treatments and procedures, such as lumbar puncture and port access. Multiple clinical studies revealed that even VR with minimal or no agency significantly attenuated cancerrelated symptoms and pain during hospitalization and during procedures. Therefore, true VR with agency would likely show an even greater pain reduction in cancer patients undergoing treatments or procedures (Indovina et al., 2018; Li et al., 2011).

Chronic pain is a common affliction and accounts partly for the prevalence of opioid prescriptions. Mallari et al. (2019) considers the findings on the impact of VR analgesia on patients with chronic pain to be less consistent than the findings on acute pain. Out of 10 studies reviewed involving patients with chronic pain, three did not reveal a decrease in pain intensity either during or after the VR exposure. A separate study assessing the impact of VR on 30 adults with various chronic pain conditions reported that pain was significantly reduced both during and immediately after the VR session (Jones et al., 2016). A non-randomized cohort study of hospitalized patients discovered that there was a larger pain reduction in the VR cohort compared to the distraction video cohort (Tashjian et al., 2017). There are mixed findings on the efficacy of VR to reduce chronic pain, possibly because of its heterogeneous origins.
In the inpatient setting, patients are suffering from nearly constant pain, whether it be acute, procedural, or chronic in nature. The overwhelming majority of clinical studies have reported promising findings indicating that VR is an effective analgesic. The remaining studies that do not show a significant VR analgesia effect indicate that VR does not exacerbate pain. All have shown negligible adverse effects from VR use.

\section{Future Directions}

Scientists have barely begun to realize the untapped potential of VR technology for applications in pain management. Historically, VR has been prohibitively expensive and required bulky equipment. As technology rapidly advances, VR has become an increasingly viable tool for pain management in hospital settings and perhaps even outpatient clinics or homes in the near future. Since the commercial introduction of VR, its associated costs have been on a steady and rapid decline, with units no longer ranging in the thousands but hundreds of dollars. Along with declining costs, VR equipment has become more compact and portable. VR technology will only continue to improve and incorporate more advances like cloud computing and artificial intelligence. In terms of its software, VR is extremely versatile; any individual can brainstorm new ideas and turn them into reality with little assistance. VR is easily customizable for the treatment, not only of a particular medical condition, but also of specific subsets of that patient demographic.

While the application of VR in clinical environments has become more tenable in the last 2 decades, there are still limitations and challenges to its implementation as a mainstream intervention. First and foremost, VR intervention is still a nascent field of research with ill-defined terminology, making it more difficult to measure outcomes. While there is no gold standard for measuring pain, an overwhelming majority of studies use various subjective pain ratings as their pain measurement tools and it is challenging to compare the reduction in pain objectively. This is further complicated by a high variance in pain thresholds across patient populations and an unknown interplay between pain and anxiety. Second, hospitals and healthcare providers might be reluctant to see VR as a worthy investment. Most people are unfamiliar with VR and do not realize the scope of its applications and potential benefits. Persuading hospital administrators that the benefits outweigh the costs of training, setup, and upkeep required for VR equipment and software use may be difficult, even with the urgency of the opioid epidemic and the rapid pace of developments in the field of VR research. Third, hygiene and sanitation are valid concerns especially in the light of the current COVID-19 pandemic. Disposable coverings on HMDs and disinfectant wipes could help mitigate these concerns.

VR holds promise to become a viable mainstream intervention and adjunct therapy to pharmacological agents, but 
further clinical research is required. Most importantly, studies need to have objective measures, like the reduction of amount of opioid administration due to adjunct VR use, so that there are quantifiable methodologies for comparing the outcomes of different studies. Most pre-existing studies involved a small number of patients, were not RCTs, and did not assess the long-term effects of VR analgesia. More RCTs with larger sample sizes and repeated VR treatments are necessary to increase the generalizability of the findings, minimize bias, and evaluate possible long-term benefits of VR analgesia. Testing different lengths of VR sessions would be crucial for determining their optimal duration.

\section{Conclusion}

Virtual reality has the potential to be a non-pharmacological adjunct that can significantly reduce the use of analgesics in hospitals. VR remains a new technology and the exact mechanism underlying its ability to reduce pain remains unknown. However, pre-existing data with predominantly subjective pain ratings as the primary measure have shown that VR intervention significantly reduces pain in patients with various medical conditions and in patients undergoing painful medical procedures. The development of VR as an effective nonpharmacological adjunct analgesic is a promising strategy to curb the ongoing public health threat posed by the opioid epidemic. It is critical that technological advances, as well as methodological advances like the objective assessment of drug sparing effects, be incorporated into future research to establish VR as a mainstream intervention for pain management in hospital settings and beyond.

Acknowledgements Special thanks to Dr Manohar Shroff, Dr Jennifer Stimec and Dr Andrea Doria at the Hospital for Sick Children for encouraging the publication of this work and inspiring us to pursue this research.

Funding This work was supported by the Department of Radiology, Lewis Katz School of Medicine, Temple University, Philadelphia, PA, USA.

Data Availability Not applicable

Code Availability Not applicable

\section{Declarations}

Competing Interests Shunn Theingi, BA, Ian Leopold, MD, and Gary $\mathrm{S}$. Cohen, MD, declare that they have no competing interests in conducting this research. Hillel S. Maresky, MD, is the chief medical officer at VRAL, and Tolulope Ola, MD, is the chair of the patient family advisory committee at VRAL.

\section{References}

Ahmadpour, N., Randall, H., Choksi, H., Gao, A., Vaughan, C., \& Poronnik, P. (2019). Virtual reality interventions for acute and chronic pain management. Int J Biochem Cell Biol, 114, 105568.

Al-Ghamdi, N. A., Meyer 3rd, W. J., Atzori, B., Alhalabi, W., Seibel, C. C., Ullman, D., \& Hoffman, H. G. (2020). Virtual reality analgesia with interactive eye tracking during brief thermal pain stimuli: A randomized controlled trial (crossover design). Frontiers in Human Neuroscience, 13, 467. https://doi.org/10.3389/fnhum. 2019.00467.

Bekelis, K., Calnan, D., Simmons, N., et al. (2017). Effect of an immersive preoperative virtual reality experience on patient reported outcomes: A randomized controlled trial. Ann Surg, 265, 10681073.

Carter, A. J., Greer, M. C., Gray, S. E., \& Ware, R. S. (2010). Mock MRI: Reducing the need for anaesthesia in children. Pediatr Radiol, 40, $1368-1374$.

Centers for Disease Control and Prevention. (2019). 2019 annual surveillance report of drug-related risks and outcomes - United States. Surveillance Special Report. Centers for Disease Control and Prevention. U.S. Department of Health and Human Services.

Centers for Disease Control and Prevention. (2021). Understanding the epidemic Opioid overdose. Centers for Disease Control and Prevention. U.S. Department of Health and Human Services. https://www.cdc.gov/drugoverdose/epidemic/index.html. Accessed 3 April 2021.

Dahlhamer, J., Lucas, J., Zelaya, C., et al. (2018). Prevalence of chronic pain and high-impact chronic pain among adults - United States, 2016. MMWR Morb Mortal Wkly Rep, 67, 1001-1006.

Florence, C. S., Zhou, C., Luo, F., \& Xu, L. (2016). The economic burden of prescription opioid overdose, abuse, and dependence in the United States, 2013. Med Care, 54(10), 901-906.

Gutierrez-Martinez, O., Gutierrez-Maldonaldo, J., Cabas-Hoyos, K., et al. (2010). Interactive and passive virtual reality distraction: Effects on presence and pain intensity. Stud Health Technol Inform, 154, 155159.

Hoffman, H. G., Patterson, D. R., \& Carrougher, G. J. (2000). Use of virtual reality for adjunctive treatment of adult burn pain during physical therapy: A controlled study. Clin J Pain, 16, 244-250.

Hoffman, H. G., Patterson, D. R., Magula, J., et al. (2004a). Waterfriendly virtual reality pain control during wound care. J Clin Psychol, 60, 189-195.

Hoffman, H. G., Richards, T. L., Coda, B., et al. (2004b). Modulation of thermal pain-related brain activity with virtual reality: Evidence from fMRI. Neuroreport, 15(8), 1245-1248. https://doi.org/10. 1097/01.wnr.0000127826.73576.91.

Hoffman, H. G., Sharar, S. R., Coda, B., et al. (2004c). Manipulating presence influences the magnitude of virtual reality analgesia. Pain, 111, 162-168.

Hoffman, H. G., Richards, T. L., Van Oostrom, T., et al. (2007). The analgesic effects of opioids and immersive virtual reality distraction: Evidence from subjective and functional brain imaging assessments. Anesthesia and Analgesia, 105(6), 1776-1783. https://doi.org/10. 1213/01.ane.0000270205.45146.db.

Indovina, P., Barone, D., Gallo, L., Chirico, A., De Pietro, G., \& Giordano, A. (2018). Virtual reality as a distraction intervention to relieve pain and distress during medical procedures: A comprehensive literature review. Clin J Pain, 34(9), 858-877.

Jones, T., Moore, T., \& Choo, J. (2016). The impact of virtual reality on chronic pain. PLoS One, 11(12), e0167523.

Kain, Z. N., Mayes, L. C., Caldwell-Andrews, A. A., Karas, D. E., \& McClain, B. C. (2006). Preoperative anxiety, postoperative pain, and behavioral recovery in young children undergoing surgery. Pediatrics, 118(2), 651-658. 
Kerns, R. D., Sellinger, J., \& Goodin, B. R. (2011). Psychological treatment of chronic pain. Annu Rev Clin Psychol, 7, 411-434.

Law, E. F., Dahlquist, L. M., Sil, S., et al. (2011). Videogame distraction using virtual reality technology for children experiencing cold pressor pain: The role of cognitive processing. J Pediatr Psychol, 36, 84-94.

Li, A., Montaño, Z., Chen, V. J., \& Gold, J. I. (2011). Virtual reality and pain management: Current trends and future directions. Pain Manag, 1(2), 147-157.

Mallari, B., Spaeth, E. K., Goh, H., \& Boyd, B. S. (2019). Virtual reality as an analgesic for acute and chronic pain in adults: A systematic review and meta-analysis. J Pain Res, 12, 2053-2085.

Melzack, R. (1999). From the gate to the neuromatrix. Pain, 6, S121S126.

Melzack, R., \& Wall, P. D. (1965). Pain mechanisms: A new theory. Science, 150, 971-979.

Mosso-Vázquez, J. L., Gao, K., Wiederhold, B. K., \& Wiederhold, M. D. (2014). Virtual reality for pain management in cardiac surgery. Cyberpsychol Behav Soc Netw, 17(6), 371-378.

Pourmand, A., Davis, S., Marchak, A., Whiteside, T., \& Sikka, N. (2018). Virtual reality as a clinical tool for pain management. Curr Pain Headache Rep, 22(8), 53.
Ryu, J. H., Park, S. J., Park, J. W., et al. (2017). Randomized clinical trial of immersive virtual reality tour of the operating theatre in children before anaesthesia. British Journal of Surgery, 104(12), 1628-1633.

Sharar, S. R., Alamdari, A., Hoffer, C., et al. (2016). Circumplex model of affect: A measure of pleasure and arousal during virtual reality distraction analgesia. Games Health J, 5, 197-202.

Tashjian, V. C., Mosadeghi, S., Howard, A. R., et al. (2017). Virtual reality for management of pain in hospitalized patients: Results of a controlled trial. JMIR Ment Health, 4(1), e9.

Triberti, S., Repetto, C., \& Riva, G. (2014). Psychological factors influencing the effectiveness of virtual reality-based analgesia: A systematic review. Cyberpsychol Behav Soc Netw, 17, 335-345.

Wender, R., Hoffman, H. G., Hunner, H. H., et al. (2009). Interactivity influences the magnitude of virtual reality analgesia. $J$ Cyber Ther Rehabil, 2, 27-33.

Won, A. S., Bailey, J., Bailenson, J., Tataru, C., Yoon, I. A., \& Golianu, B. (2017). Immersive virtual reality for pediatric pain. Children (Basel), 4(7).

Publisher's Note Springer Nature remains neutral with regard to jurisdictional claims in published maps and institutional affiliations. 